\title{
Valorización del uso de la lengua rapanui
}

\author{
Valorization of the use of rapanui language
}

\author{
Mattarena Haoa \\ Departamento de Antropología, Universidad de Chile \\ elcieloestaestrellado@hotmail.com
}

\begin{abstract}
Resumen
El estudio realizado está orientado en la relevancia que implica el uso de la lengua rapanui. La indagación ha sido realizada a través de una muestra de 32 casos en residentes de la localidad de Rapa-Nui durante los meses de Abril y Mayo de 2017. Los resultados indican que existe una preocupación entre los encuestados por el detrimento en el uso del idioma rapanui y por ende la irremediable pérdida de los principales medios de transmisión de la "memoria biocultural". La falta de consideración de la lengua rapanui, ha sido provocada por distintas causas, las que se relacionan con la ilusión de una carencia funcional a nivel de desarrollo de la misma comunidad. Esto ha sido provocado, no solo por el fenómeno que implica la globalización, sino también por diversas entidades que no ven en el uso de la lengua rapanui, una herramienta que potencie sus actividades e intereses, entre ellas podemos mencionar: las instituciones gubernamentales, organizaciones turísticas y la masiva inmigración hispanohablante. Así es que, familias completas han fomentado el uso de la lengua castellana en desmedro del rapanui, sin ser conscientes de llevar toda una cosmovisión a su sepultura.
\end{abstract}

Palabras clave: lengua, rapanui, globalización, pérdida, cosmovisión.

\begin{abstract}
The study developed, is oriented in the relevance that implies the use of rapanui language. The investigation was produced through a sample of 32 cases in residents of the town of Rapa-Nui during the months of April and May of 2017. The results indicate that there is a concern among the respondents for the detriment in the use of the rapanui language and therefore the irremediable loss of the main means of transmission of the "biocultural memory". The lack of consideration of the rapanui language has been occasioned by different causes, which are related to the illusion of a functional deficiency at the development level of the community itself. This has been caused, not only by the phenomenon that globalization implies, but also by various entities that do not see the use of the rapanui language, a tool that enhances their activities and interests, among them we can mention: governmental institutions, touristic organizations and the massive spanish-speaking immigration. Thus, whole families have encouraged the use of the castilian language to the detriment of rapanui, without being aware of bringing a whole worldview to his grave.
\end{abstract}

Key words: language, rapanui, globalization, loss, worldview. 


\section{Introducción}

La presente investigación, refiere al proceso de difusión de la lengua rapanui en los hogares y adicionalmente a la recepción de aquellos a quienes se les transmite.

El propósito de este análisis, sugiere la reunión de información que nos permita relacionar en base a la procedencia de hogares con familia rapanui y su correspondencia con el uso de la lengua vernácula en diversos espacios, tales como: centros educacionales, espacios domésticos y zonas laborales y de esparcimiento.

La implementación de la educación formal en Rapa-Nui en 1934, no consideró el idioma rapanui dentro de los contenidos del currículum educativo, a pesar del $77 \%$ de jóvenes de habla rapanui en ese momento. Las dificultades en el proceso de aprendizaje impartido en habla hispana causaron grandes estragos a nivel social, cultural e incluso espiritual, situación que no se revirtió hasta que se inició la introducción de la lengua vernácula en la única escuela existente en Rapa Nui, en ese momento. Hasta entonces, la lengua rapanui fue más bien silenciada dentro de la educación formal, como una manera de sumergir a los estudiantes en los contenidos por medio de un sistema de aprendizaje español efectivo, pero descontextualizado.

El objetivo de esta incursión pretende: revitalizar, conservar y transmitir el idioma rapanui a las nuevas generaciones. La estructura del artículo se enmarca en un contexto mayor que implica aspectos como los de cultura y lenguaje, aludiendo a su relación en las formas de ver y organizar el mundo, lo cual comprende no solo a las personas, sino también al modo en que perciben la realidad. El lenguaje, es un instrumento que estructura e interpreta las percepciones externas e internas del pensamiento en palabras, lo que permite construir la realidad. Por lo tanto, cambiar ese instrumento, altera también nuestra realidad generando problemas sociales, culturales e incluso espirituales.

\section{Metodología}

Se realizó una encuesta a través de Google formularios, la cual fue enviada a través de whatsapp y correo electrónico. La encuesta fue compartida a través de un muestreo tipo bola de nieve, durante los meses de Abril y Mayo del año 2017. Se respondieron 32 encuestas, de las que 4 casos no fueron contestadas totalmente. El sondeo se realizó en base a seis interrogantes de selección múltiple para continuar con tres preguntas de desarrollo, donde los encuestados manifestaron sus opiniones sobre el uso de la lengua rapanui más una pregunta abierta sobre la forma de abordar la transmisión de esta.

A modo de ampliar la pesquisa, hemos incluído un soporte documental proveniente del portal de YouTube titulado: "Rapa Nui, el ombligo del mundo". El recurso audiovisual presenta comentarios realizados por visitantes de la página (extranjeros y chilenos) donde manifiestan diversas apreciaciones, las que nos permiten distinguir algunos criterios utilizados por quienes las emiten.

La investigación fue realizada por medio de distintos recursos, los que contribuyeron en la compilación y selección de información a través de nodos, los que actúan como depósitos de información con 
características semejantes. Un ejemplo es el nodo ser rapanui, el cual contiene opiniones de los encuestados que manifiestan rasgos como la pérdida del habla, la pérdida de identidad, la pérdida de cultura y la falta de entendimiento. La información fue codificada de acuerdo a las declaraciones de los encuestados, las que fueron previamente identificadas a partir de sus opiniones, para posteriormente ser ingresadas en los distintos nodos conforme a su pertinencia. Una vez realizados los pasos mencionados, se realizó el procesamiento de la información, mediante el análisis de las declaraciones recogidas para luego ser expresadas a través de modelos gráficos que permitiesen expresar visualmente, lo reflejado en las opiniones.

\section{Análisis de la información}

\section{Relación entre provenir de familia rapanui y el uso del idioma}

De acuerdo a los datos obtenidos podemos interpretar que pertenecer a una familia rapanui no es imprescindible para hablar la lengua $y$, en el caso de integrarla, no equivale a hablar el idioma, por lo tanto, el uso de la lengua no depende exclusivamente de la procedencia familiar, sino más bien de un interés proveniente de la misma persona y su relación con quienes lo hablen.

\section{Gráfico 1}

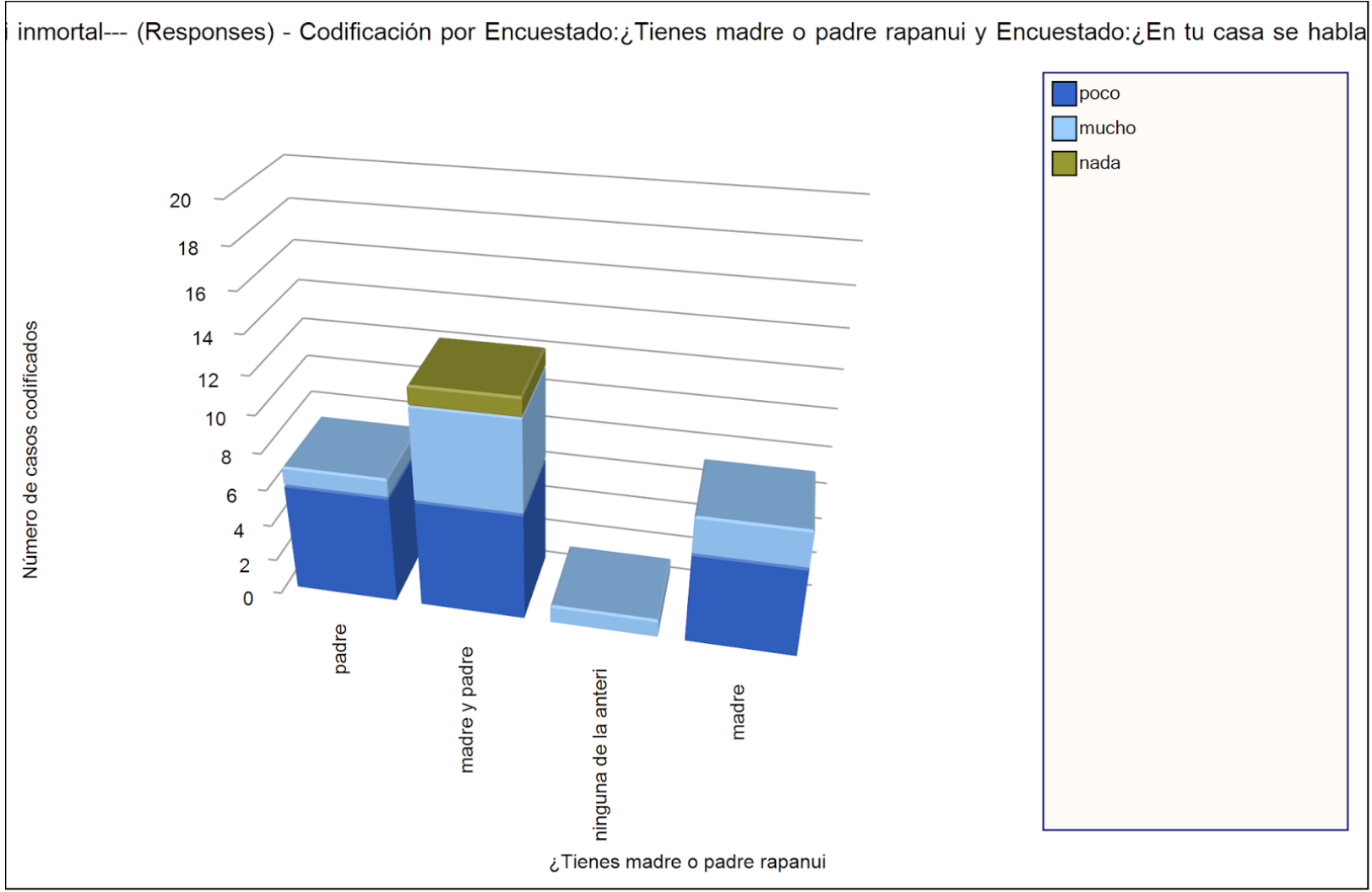




\section{Relación entre hablar rapanui en casa y hablar rapanui con los amigos}

De los casos analizados podemos inferir que hablar rapanui en el hogar, no determina que se hable el idioma con los amigos y así a la inversa. De acuerdo a lo indicado anteriormente, es admisible suponer que el uso del idioma, se vincula más bien con una inclinación de la persona a hablar que con la filiación a un hogar rapanui. Así también, podemos señalar que otros ambientes, distintos a los familiares, pueden actuar como espacios de inspiración y reproducción del idioma.

\section{Gráfico 2}

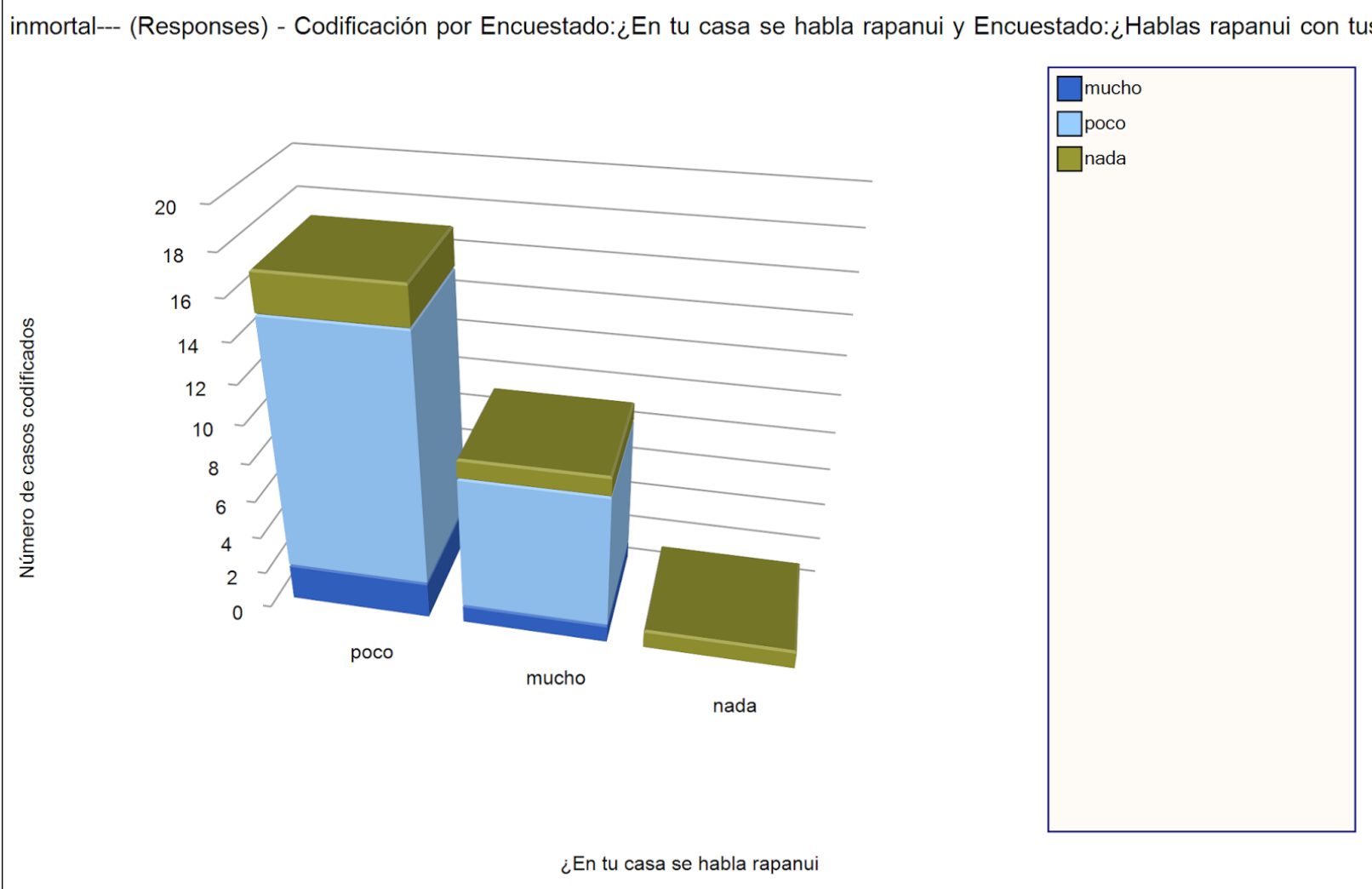

\section{Relación entre madre que habla rapanui y el uso del idioma en el trabajo o colegio}

Es posible considerar que no existe una correlación evidente entre madres que hablan rapanui y el uso del idioma en espacios educativos y/o laborales. De acuerdo a los antecedentes recopilados podemos deducir que en varios casos donde las madres hablan rapanui de manera fluida, los hijos se expresan escasamente en rapanui dentro de sus trabajos o colegios.

Algunos casos presentan madres no hablantes de la lengua, sin embargo los hijos se relacionan en rapanui de manera limitada.

Existen solo dos casos donde las madres no hablan el idioma y sus hijos tampoco lo hacen en las zonas donde trabajan o estudian. 
Se afirma que no existe completa correspondencia entre descender de hogares con madres rapanui parlantes y el despliegue del idioma de los individuos en espacios públicos.

\section{Gráfico 3}

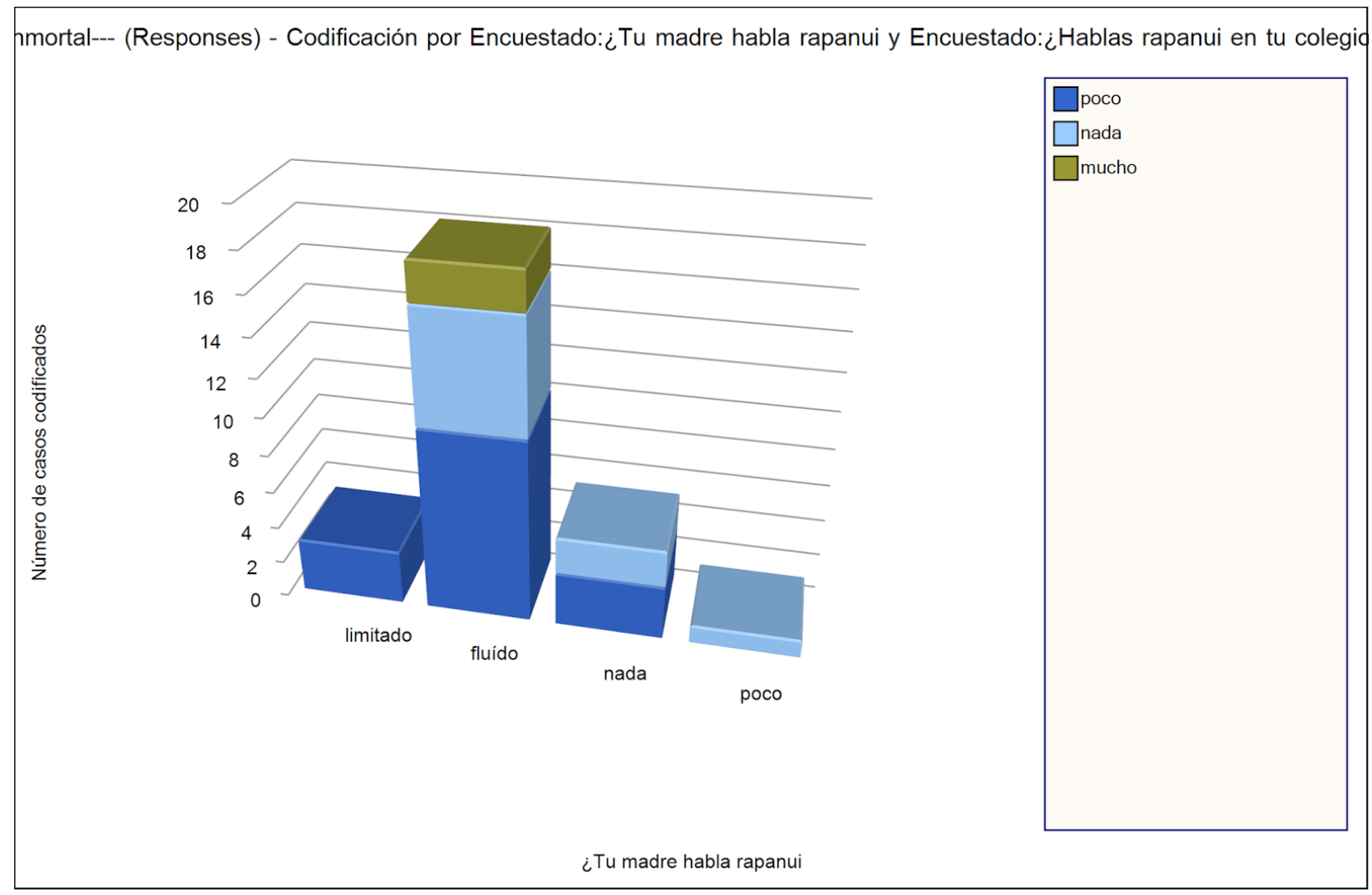

\section{Relación entre madres que hablan rapanui y el uso del idioma en casa}

De las referencias observadas, podemos dilucidar la existencia de una relación entre madres que hablan rapanui y la reproducción del idioma por parte de sus hijos dentro de sus hogares, incluso si en algunos casos existan madres que no hablen la lengua. En conclusión, podemos presumir que las madres rapanui parlantes enfatizan el uso de la lengua dentro del hogar, lo que estimula a sus hijos a imitarlas. 


\section{Gráfico 4}

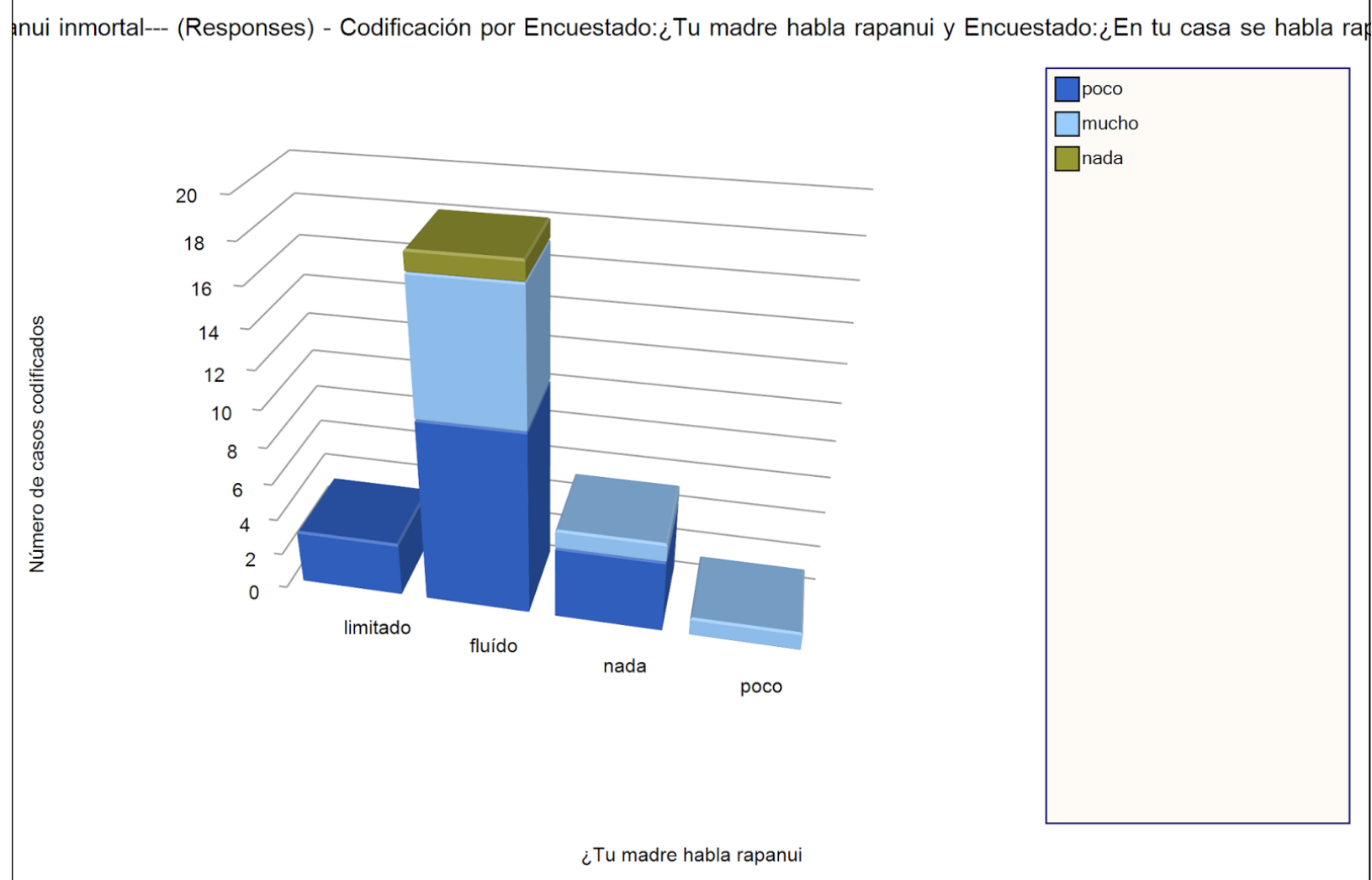

\section{Relación entre padre que habla rapanui y el uso del idioma en casa}

Conforme a los datos codificados podemos establecer que no existe un vínculo evidente entre los padres que hablan rapanui y la reproducción del idioma en el hogar por parte de los hijos, por lo que podemos inferir que existe la posibilidad de que los padres no enfaticen la instrucción y mantención de la lengua. De esto se desprende que, aunque el padre domine la lengua, no es concluyente para que sus hijos hagan uso del idioma en sus hogares. 


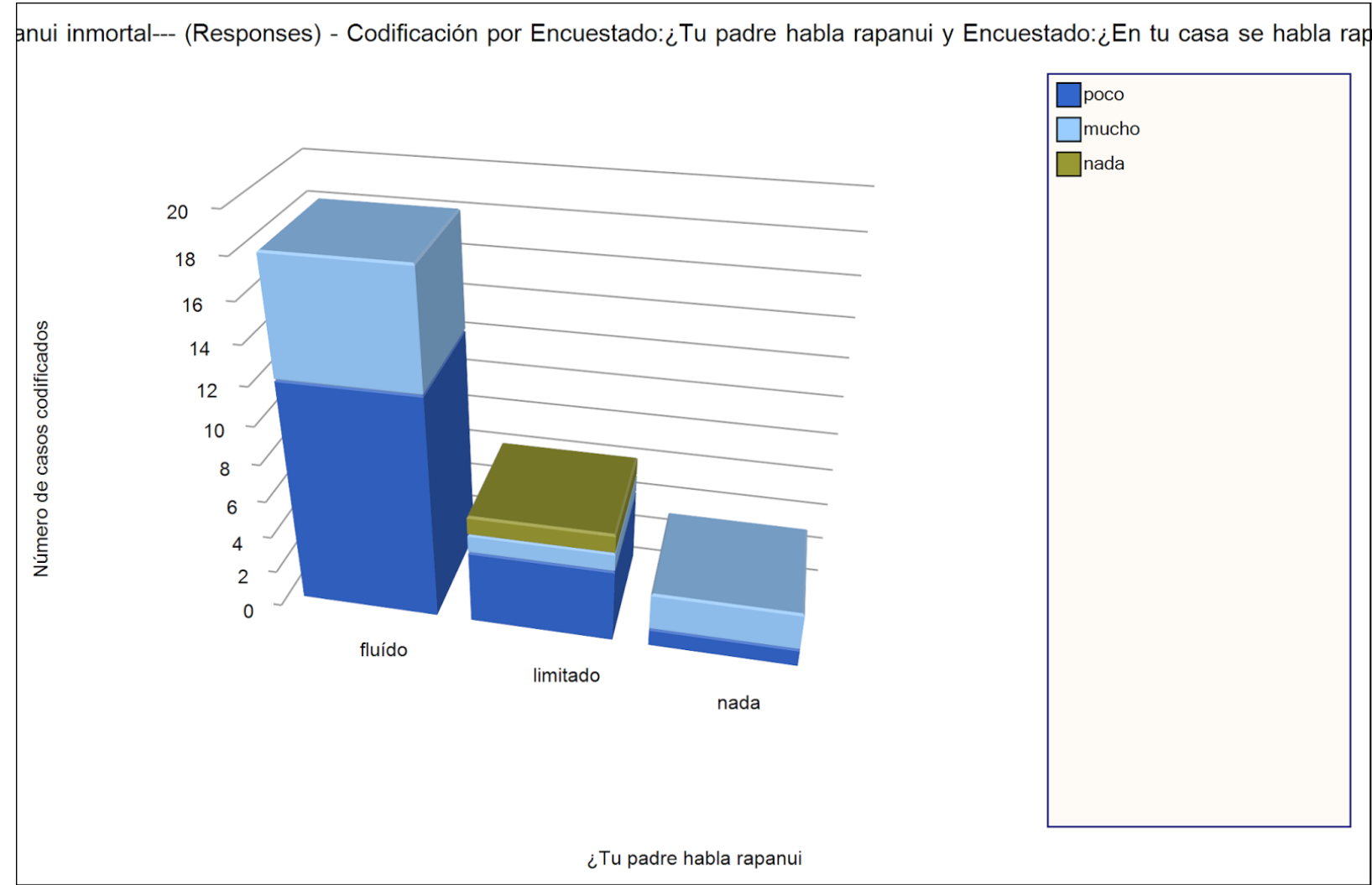

\section{Análisis de categorías}

La categoría 1 concerniente al Nodo "ser rapanui", corresponde al juicio emitido por los encuestados sobre el riesgo que implica el hecho de no hablar la lengua rapanui. Es posible advertir entre las personas consultadas que existe una preocupación latente, asociado a la pérdida del idioma.

El uso de la lengua rapanui constituye parte del sistema hereditario de los encuestados, quienes manifiestan su intranquilidad, pues comprenden que existen graves consecuencias sujetas al abandono de la lengua vernácula en perjuicio de la identidad.

La categoría 2 corresponde al Nodo "propuesta", el cual sugiere distintas estrategias, recomendadas por los encuestados para fomentar la revalorización de la lengua. Los métodos mencionados refieren a diversos procedimientos con intención de estimular el uso y la preservación del idioma.

Las propuestas ofrecidas implican promover el idioma a través de distintos ámbitos de los que podemos nombrar: el uso obligatorio de la lengua rapanui al interior de establecimientos educacionales, además de la promoción del idioma a través de distintos medios de comunicación como por ejemplo: programas en rapanui a través de la radio y la televisión, así como la producción de textos impresos y talleres dirigidos por personas ilustradas en el tema que orienten en la instrucción y práctica del idioma en zonas laborales, domésticas o de esparcimiento. 
La categoría 3 pertenece al Nodo "áreas afectadas", la cual refiere a la trascendencia del uso del idioma rapanui y cómo esto afecta la cotideaneidad de los encuestados. Los resultados permiten evidenciar una imagen crítica sobre el presente del idioma rapanui, provocado por el desplazamiento de la lengua castellana en el pasado y las oportunidades que ésta ofrecía, de trabajo, educación y comunicación. Esto provocó que la transmisión de la lengua rapanui, en muchos hogares se tornara irrelevante, por ser percibida como un obstáculo potencial para el éxito en la vida; no obstante, los encuestados enfatizan su importancia hoy en día al advertir los daños irreparables que implicaría la pérdida del idioma.

La categoría 4 se encuentra asociada al Nodo "opiniones", este apartado considera la grabación de un corto titulado "Rapa Nui, el ombligo del mundo" a modo de ampliar las fuentes utilizadas dentro de la investigación. Los comentarios realizados al vídeo, exponen las apreciaciones de los visitantes de la página sobre Rapa Nui y otras áreas como la música, el turismo y la política.

\section{Conclusión}

La lengua rapanui ha sido devaluada por no presentar una funcionalidad significativa para el desarrollo de su comunidad, donde las instituciones estatales solo consideran el habla hispana, así como también los entes turísticos actuales. La inmigración hispanohablante también se ha incrementado considerablemente en las últimas décadas $\mathrm{y}$ todo esto ha derivado en importantes cambios socioculturales dentro de la misma comunidad. Las familias han optado por alternativas opuestas a las propias, con intención de proyectar una "mejor calidad de vida" para sus hijos e hijas; sin ser conscientes de que la lengua materna, remite a lo que concierne al alma, a los sentimientos, a las relaciones con otras personas y con su ecosistema. Esto permite una visión de mundo diferente, la cual posibilita no solo enriquecer, sino también enaltecer a otras culturas.

La falta de consideración a nivel transversal en los estamentos de la comunidad, podría provocar el desuso de la lengua rapanui, así como ha ocurrido con muchas otras lenguas que se han extinguido por falta de conciencia y lealtad. Es imprescindible la pronta reparación, que permita que el idioma rapanui no ocupe un plano secundario al español ni a otro idioma, sino más bien que se le atribuya la misma importancia a través de la apertura y crecimiento de neologismos, los que contienen no solo a la tecnología, sino también otros saberes.

Resulta indispensable reflexionar sobre la salud del idioma polinésico rapa nui, pues este conlleva fenómenos y efectos irreversibles producidos por la globalización, introduciendo secuelas directas sobre las cosmovisiones de los pueblos, sobre su comportamiento con el ecosistema y en la propia lingüística. 
Anexos: Nubes de palabras de cada categoría de análisis

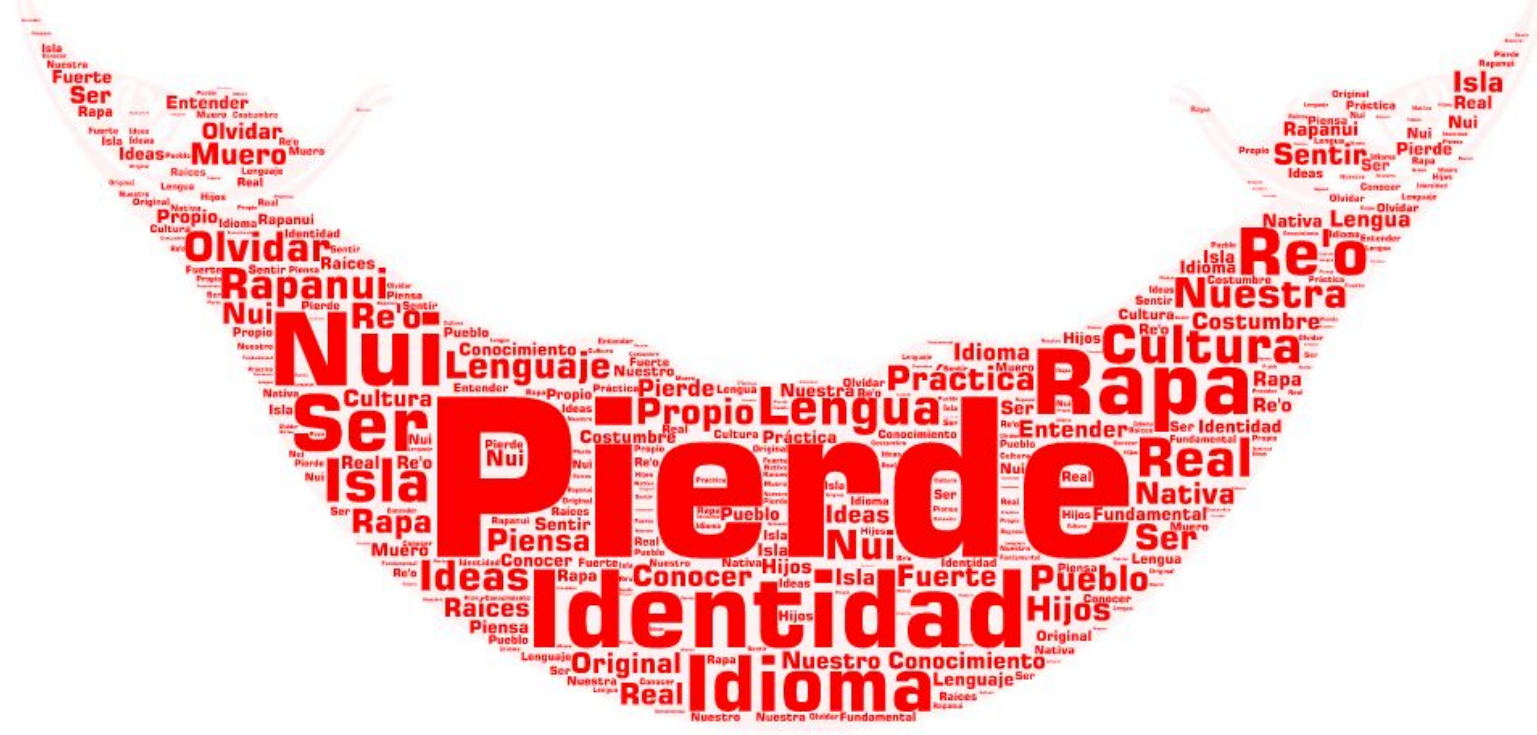




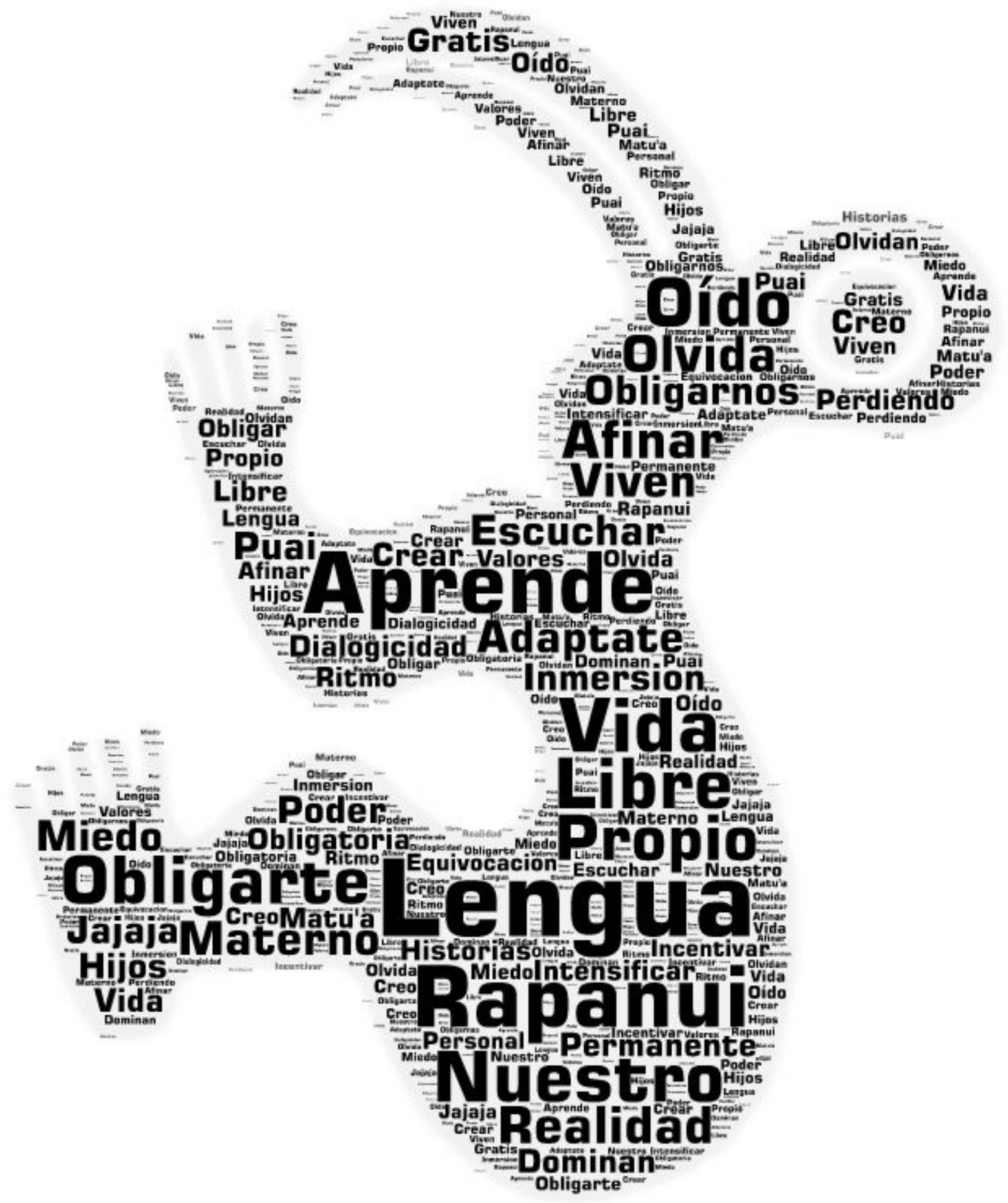



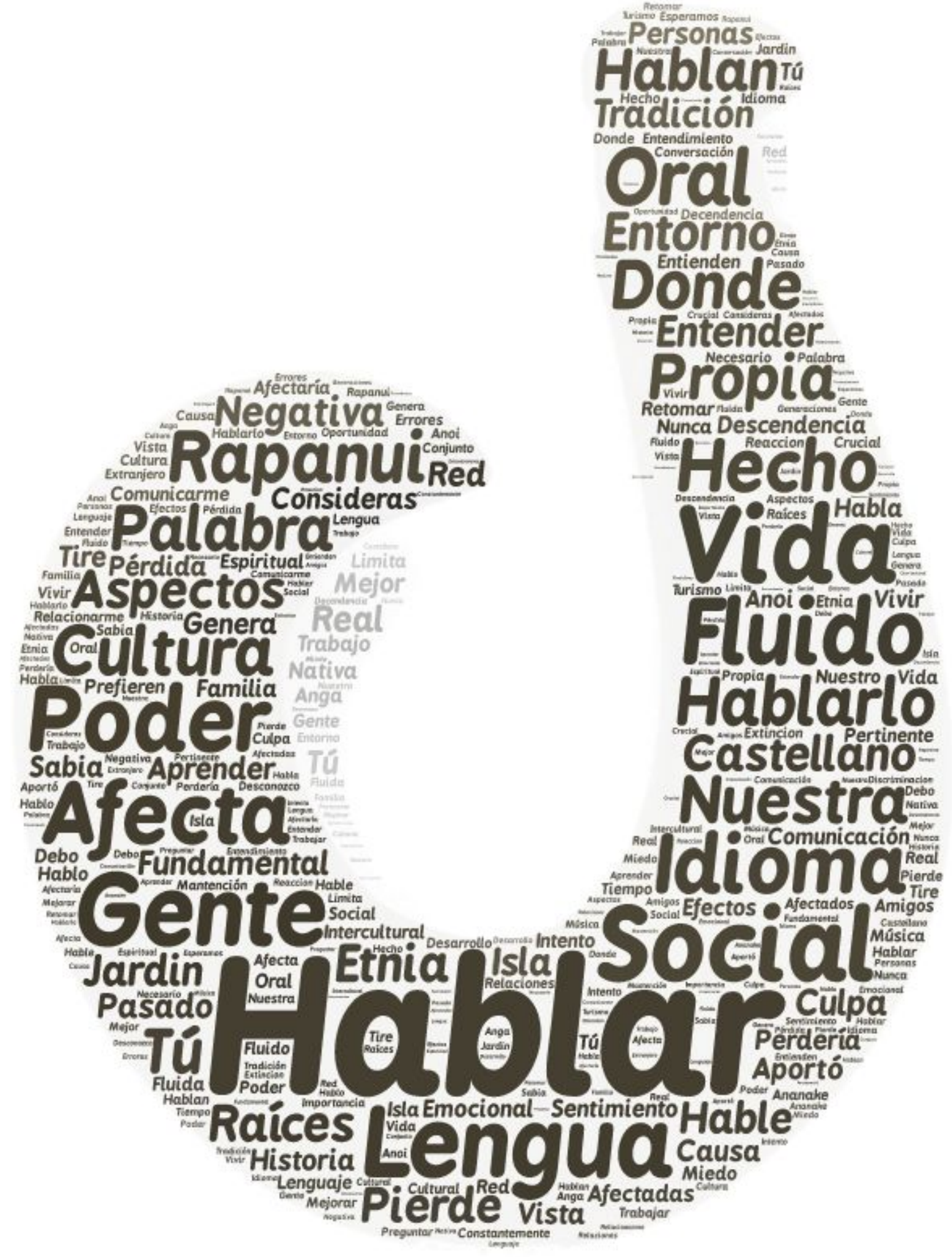

Entender

D Necesario Palabro

Numar Descendencia

midto - Reaccion Crucia

niñ Oraices Habla

Consideras

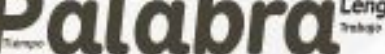

Tire pérdida Espiritual

Nenera

oral

Nativa

Prefieren Familia Angd

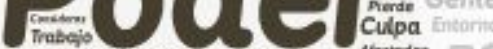

Sabia

Debo Debo

Hablo Fundamental

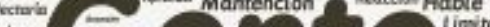

anar a $a$ Social

Jardin Oral

Etnia

ivir

(a)

Hablarto castellano=

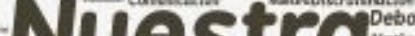



